\title{
iMixPro: intelligent Mixing of Proteomes for elimination of false positives in affinity purification-mass spectrometry
}

Sven Eyckerman ${ }^{1,2}$, Francis Impens ${ }^{1,2}$, Emmy Van Quickelberghe ${ }^{1,2}$, Noortje Samyn ${ }^{1,2}$, Giel Vandemoortele $^{1,2}$, Delphine De Sutter ${ }^{1,2}$, Jan Tavernier ${ }^{1,2}$, Kris Gevaert ${ }^{1,2}$

${ }^{1}$ VIB Medical Biotechnology Center, VIB, A. Baertsoenkaai 3 B-9000 Ghent, Belgium

${ }^{2}$ Department of Biochemistry, Ghent University, A. Baertsoenkaai 3 B-9000 Ghent, Belgium

\section{Supplementary Information}

Supplementary Figure 1: Ratio histograms for identified and quantified proteins for RNF41 and TANK experiments.

Supplementary Figure 2: Ratio histograms for identified and quantified peptides for RNF41 and TANK experiments.

Supplementary Figure 3: Ratio histograms for identified and quantified proteins and peptides for a TANK repeat experiment.

Supplementary Figure 4: Scatter plot showing the $\mathrm{H} / \mathrm{L}$ ratios in a $\log 2$ scale for peptides identified in both repeats.

Supplementary Figure 5: Histogram plots for the protein ratios obtained after iMixPro AP-MS for endogenous PTPN14 and IQGAP1.

Supplementary Figure 6: Histogram plots for the peptide ratios for endogenous PTPN14 and IQGAP1.

Supplementary Table 1: Relevant statistics for the iMixPro AP-MS experiments performed in this study showing the number of MS/MS spectra recorded, the number of peptide to spectra matches (PSMs) or identified MS/MS spectra, the number of peptides and quantified peptides, and the associated number of proteins and quantified proteins.

Supplementary Table 2: All proteins identified and quantified with consistent Heavy over Light (H/L) peptide log2 ratios higher than -1 .

Supplementary Table 3: SAINT analysis for the AP-MS experiments performed for RNF41 and TANK. 


\section{Supplementary Figures}
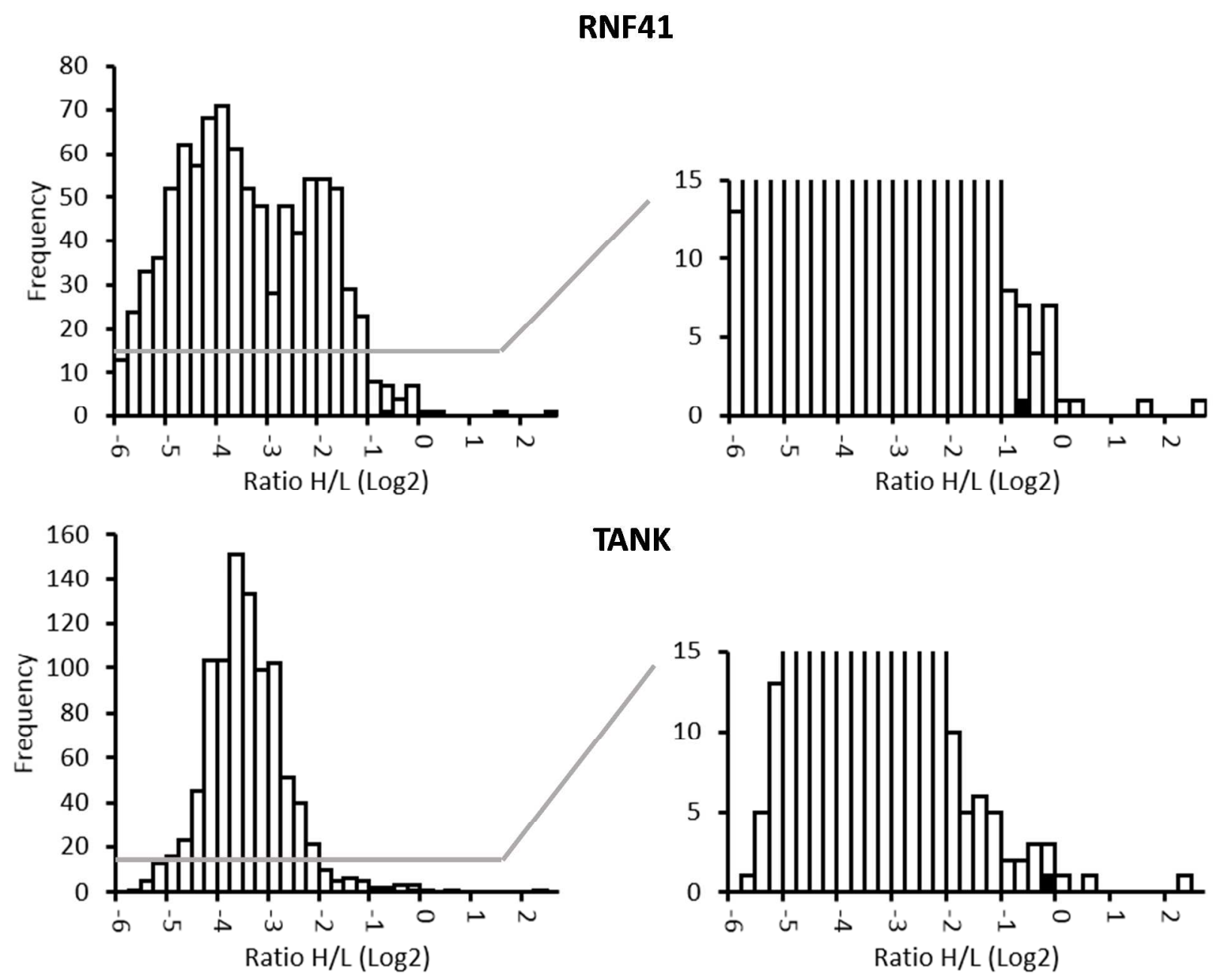

TANK

Supplementary Figure 1: Ratio histograms for identified and quantified proteins for RNF41 (upper panels) and TANK (lower panels). The bait proteins are highlighted in black (right panels for zoomed regions). The heavy $\mathrm{H} / \mathrm{L}$ ratios on the $\mathrm{X}$-axis are shown in a log2 scale. 


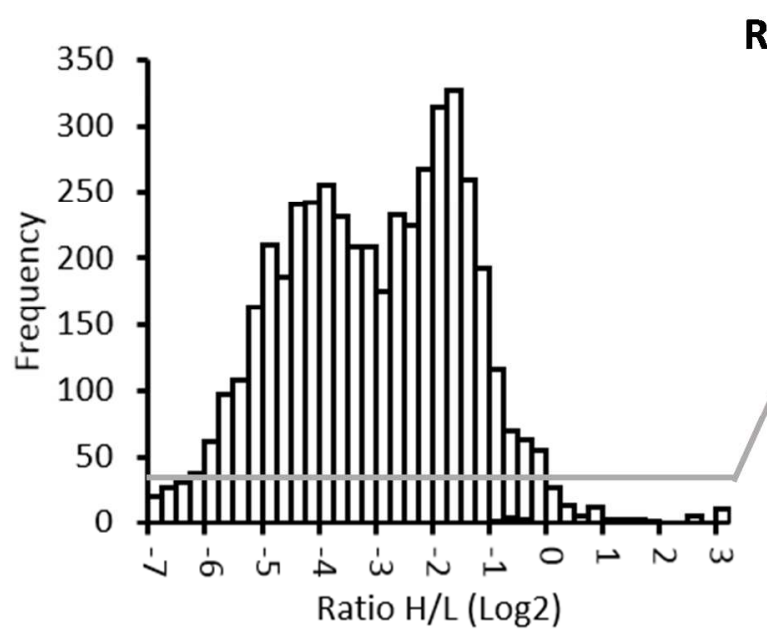

RNF41
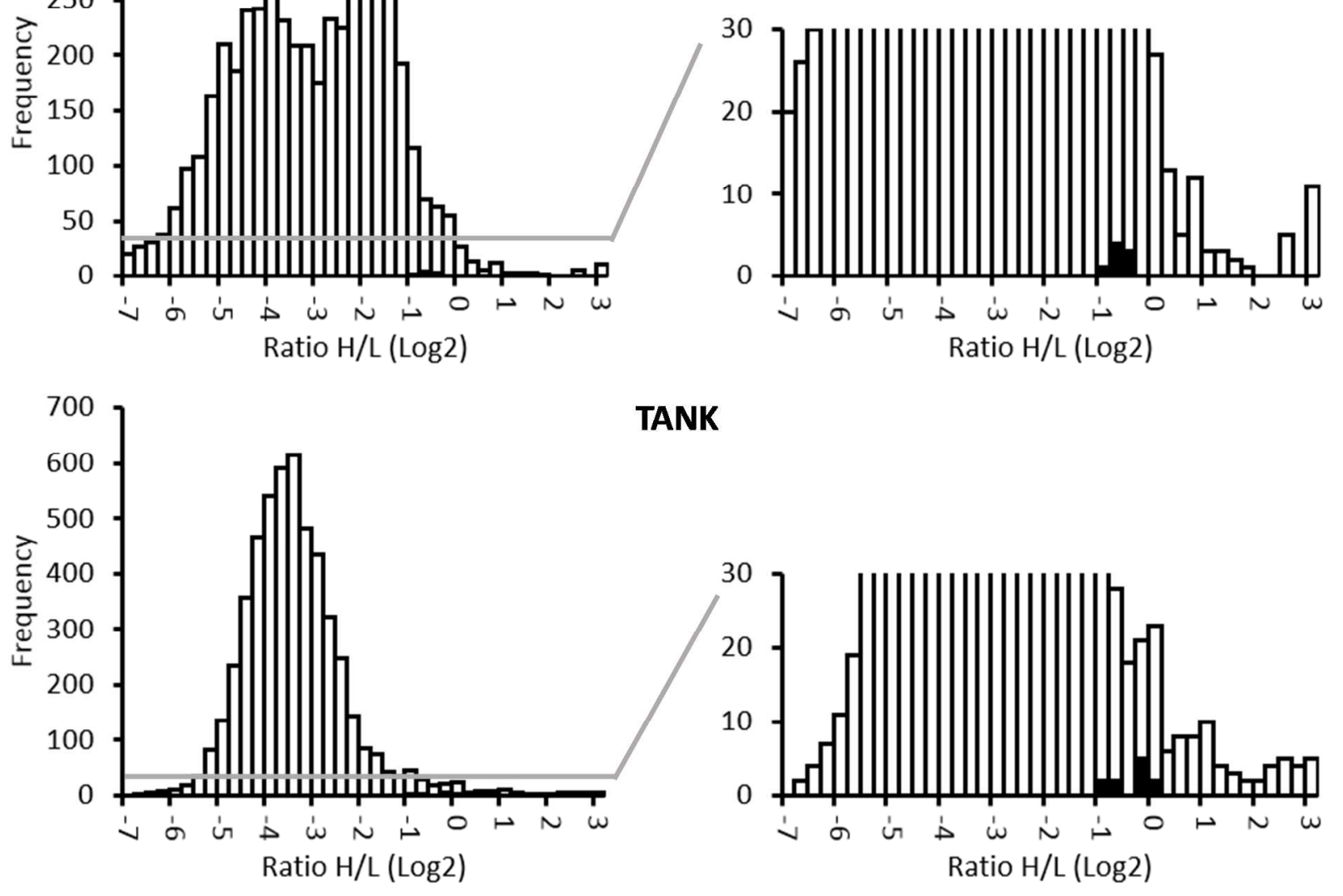

TANK

Supplementary Figure 2: Ratio histograms for identified and quantified peptides for RNF41 (upper panels) and TANK (lower panels). The bait peptides are highlighted in black (Right panels for zoomed regions). The heavy $\mathrm{H} / \mathrm{L}$ ratios on the $\mathrm{X}$-axis are shown in a log2 scale. 


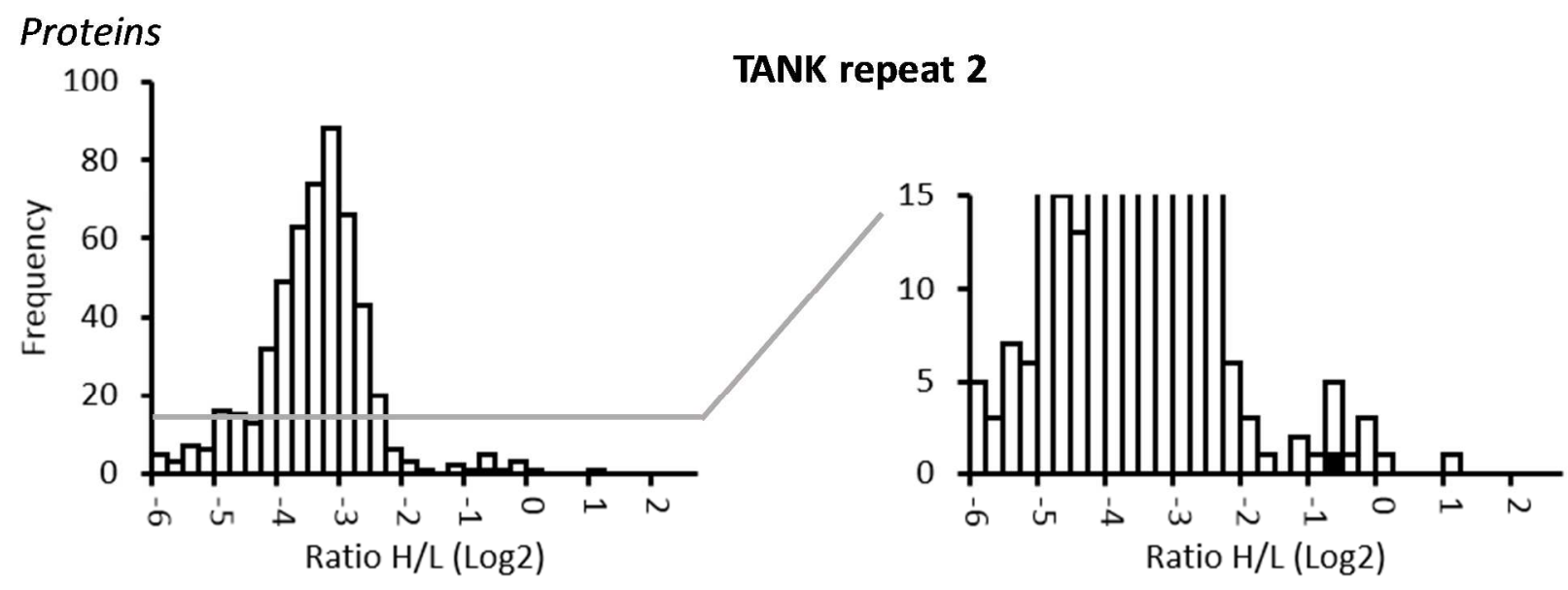

Peptides

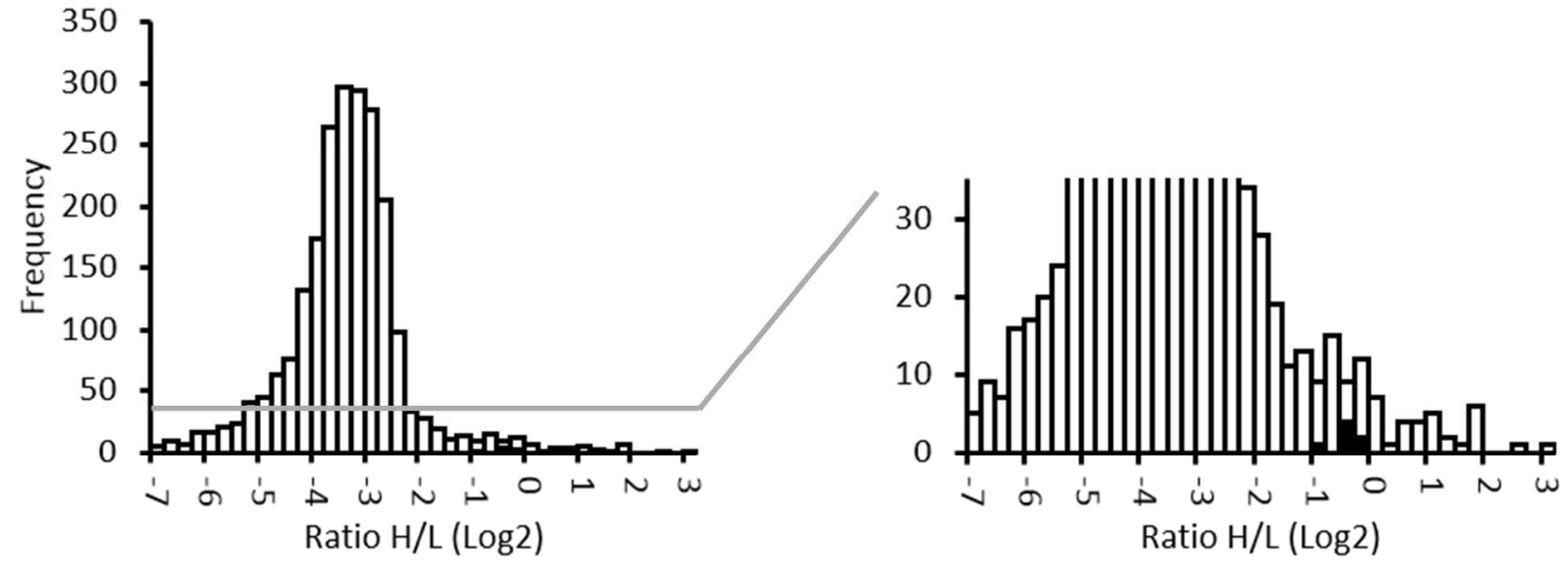

Supplementary Figure 3: Ratio histograms for identified and quantified proteins (upper) and peptides (lower panels) for a TANK repeat experiment. The heavy over light $(\mathrm{H} / \mathrm{L})$ ratios on the $\mathrm{X}$-axis are shown in a $\log 2$ scale. 


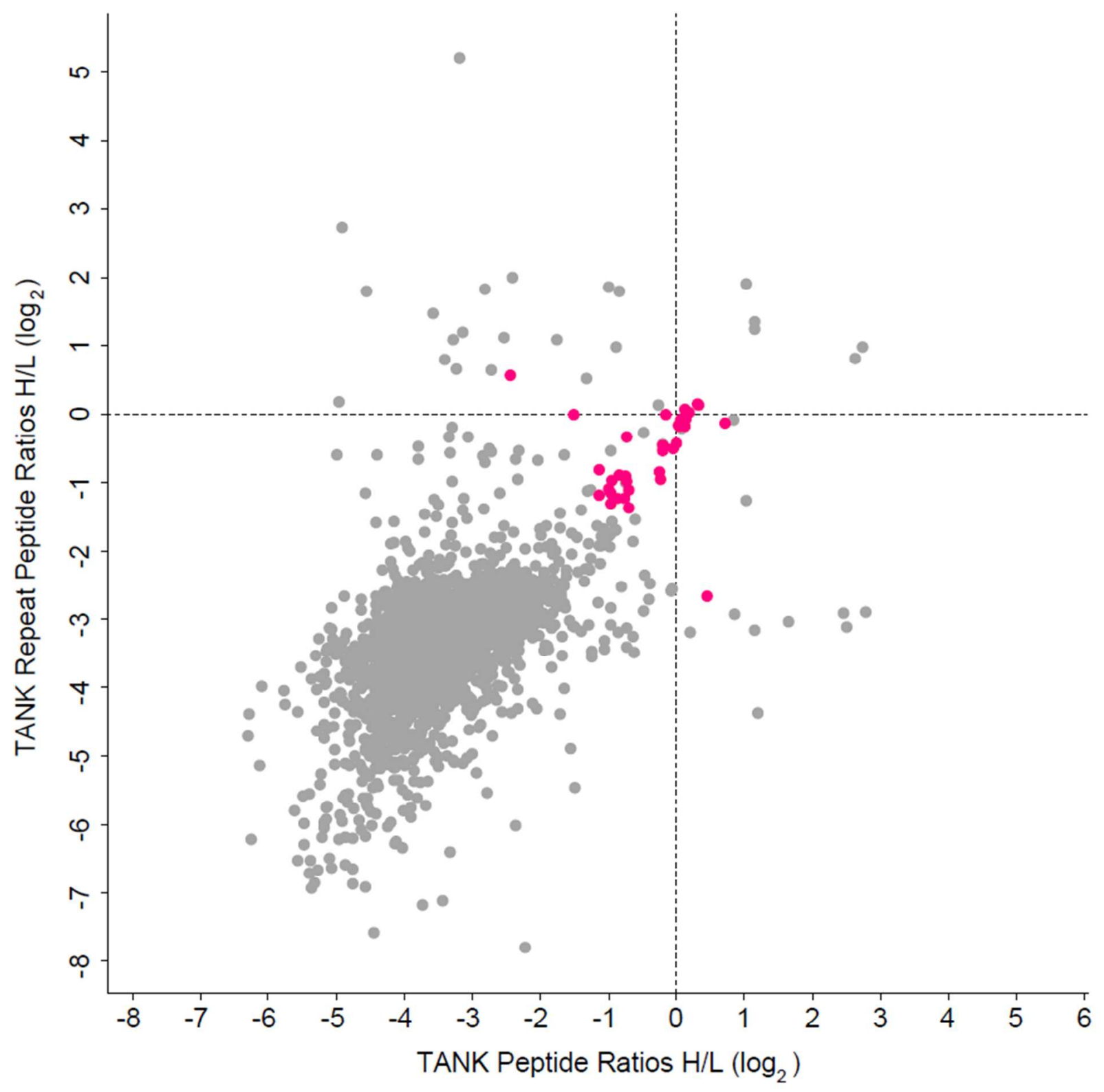

Supplementary Figure 4: Scatter plot showing the $\mathrm{H} / \mathrm{L}$ ratios in a $\log 2$ scale for peptides identified in both repeats (Pearson correlation coefficient $r=0.558$ ). The peptide ratios for TANK and for its protein partners (BIRC2, TBK1, TRAF1, TRAF2, TRAF3 and DIABLO) are shown in red. 

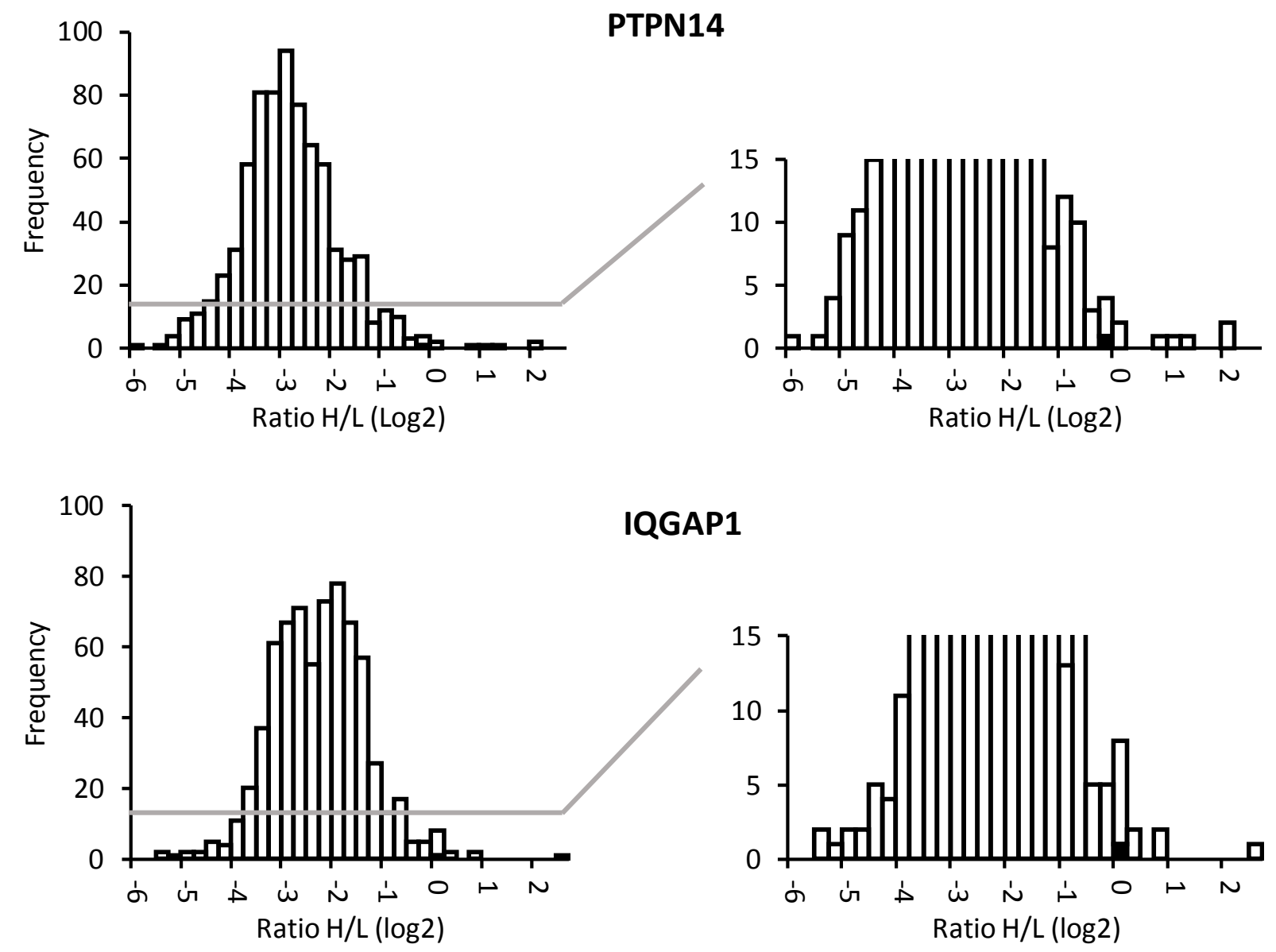

Supplementary Figure 5: Histogram plots for the protein ratios obtained after iMixPro AP-MS for endogenous PTPN14 (upper), and IQGAP1 (lower panels). The bait proteins are highlighted in black (right panels for zoomed regions). The heavy $\mathrm{H} / \mathrm{L}$ ratios on the $\mathrm{X}$-axis are shown in a log2 scale. 


\section{PTPN14}
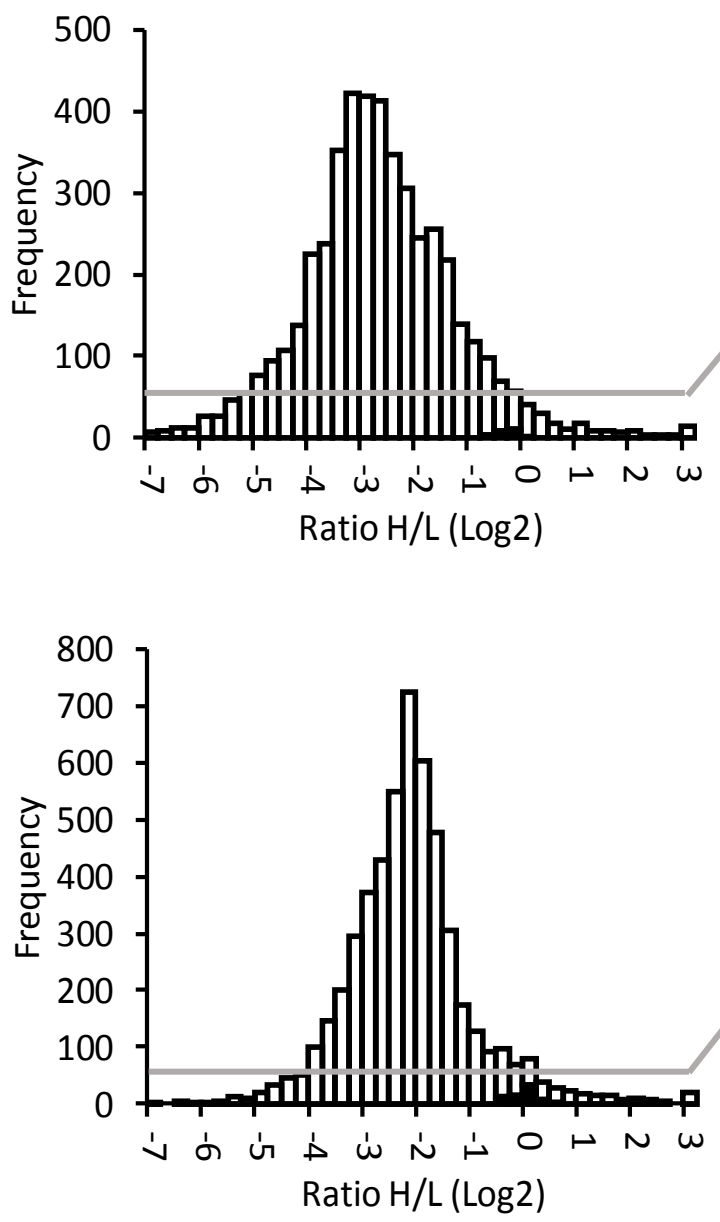

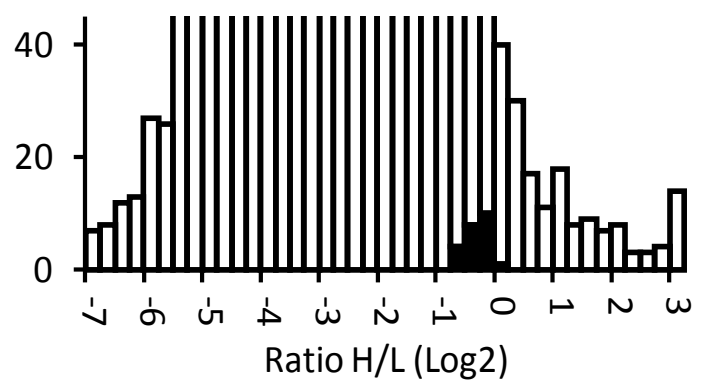

IQGAP1

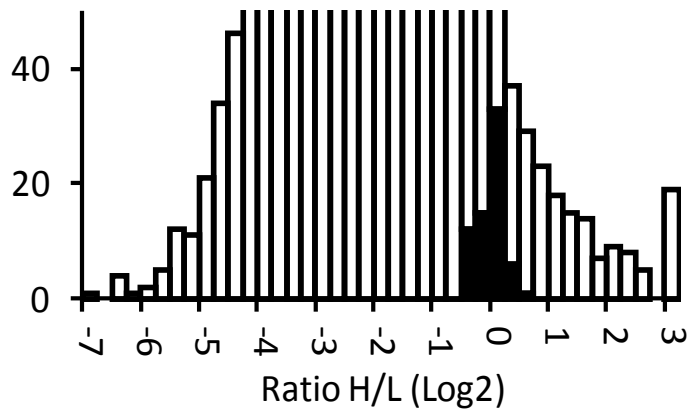

Supplementary Figure 6: Histogram plots for the peptide ratios for endogenous PTPN14 (upper panels), and IQGAP1 (lower panels). The PTPN14 and IQGAP1 bait peptides are highlighted in black (Right panels for zoomed regions). The heavy over light $\mathrm{H} / \mathrm{L}$ ratios on the $\mathrm{X}$-axis are shown in a log2 scale. 


\section{Supplementary Tables}

Supplementary table 1: Relevant statistics for the iMixPro AP-MS experiments performed in this study showing the number of MS/MS spectra recorded, the number of peptide to spectra matches (PSMs) or identified MS/MS spectra, the number of peptides and quantified peptides, and the associated number of proteins and quantified proteins.

\begin{tabular}{lllllll} 
Experiment & $\begin{array}{l}\text { \# MS/MS } \\
\text { spectra }\end{array}$ & \#PSMs & \#Peptides & $\begin{array}{l}\text { \#Quantified } \\
\text { Peptides }\end{array}$ & \#Proteins & $\begin{array}{l}\text { \#Quantified } \\
\text { Proteins }\end{array}$ \\
\hline RAF1 & 63865 & 10645 & 4408 & 3866 & 1118 & 677 \\
\hline RNF41 & 85025 & 13473 & 6278 & 4742 & 1819 & 963 \\
\hline TANK & 63170 & 11494 & 6179 & 5160 & 1465 & 945 \\
\hline TANK R & 20144 & 3146 & 6179 & 2250 & 1465 & 537 \\
\hline PTPN14 & 31142 & 5620 & 5515 & 4734 & 1188 & 745 \\
\hline JIP3 & 47404 & 7646 & 10356 & 2888 & 1929 & 515 \\
\hline IQGAP1 & 53698 & 12735 & 8611 & 5214 & 1478 & 693 \\
\hline
\end{tabular}

